\title{
A Service-oriented Architecture Framework for Emergency Vehicles Information System
}

\author{
Guangliang $\mathrm{LI}^{\mathrm{a}, *}$, Minghai $\mathrm{YAO}^{\mathrm{b}}$, Liping TANG ${ }^{\mathrm{b}}$, Qinlong $\mathrm{GU}^{\mathrm{b}}$, Hua ZHU ${ }^{\mathrm{b}}$ \\ ${ }^{a}$ College of Computer Science and Technology, Zhejiang University of Technology, Hangzhou, China \\ ${ }^{b}$ College of Information Engineering, Zhejiang University of Technology, Hangzhou, China
}

\begin{abstract}
Using Service-Oriented Architecture (SOA) to build an information system is a hot topic at present. The primary goal of this paper is to investigate how to build a SOA-based information system in a special industrythe field of emergency vehicles in environment protection industry. The paper provides a presentation of current compositions of the emergency vehicles information system and then gives out the system structure. The paper proceeds with the business process flow and working principle of an emergency vehicle information system. A service-oriented architecture framework for emergency vehicles information system is proposed.
\end{abstract}

Index Terms: Service-oriented Architecture; Emergency Vehicles; Web Service; System Framework

(C) 2011 Published by MECS Publisher. Selection and/or peer review under responsibility of the Research Association of Modern Education and Computer Science.

\section{Introduction}

Along with the rapid development of Cloud Computing, SOC (Service-Oriented Computing) and SOA (Service-Oriented Architecture) are both highly valued by most of the IT companies and institutions for academic research more and more, also they get unprecedented development in the field of software engineering. Compared with other distributed computing technology, such as Microsoft's DCOM, SOA is not completely a new one in this field [1]. However, SOA is the most important one of the foundations of Cloud Computing's basic concept and support technology, and it represents the new generation of distributed computing platform. SOA is a new kind of computing paradigm, which include new concepts, theories, and technical framework [2].

In the field of software engineering, SOA has been widely applied and is an ideal software architecture design pattern in most professions. At present, however, the system applications based on SOA are limited in the fixed fields, such as banking, logistics, communications, electric power, etc. The purpose of this article is to provide a SOA-based solution for the Emergency Vehicle Information System (EVIS) in the environment protection field. First, the paper introduces the summary of SOA and its application in software engineering is discussed. Then, the current situation of the emergency vehicles information system which is gradually

* Corresponding author:

E-mail address: liguangliang-163@163.com 
implemented in the environment protection field is studied. We give out the problems of these systems. Emphatically, we propose the design scheme based on SOA of the emergency vehicles information system. Combining the business process flow, we studied the services' design and the using method of the services.

\section{Service-Oriented Architecture}

The W3C (www.w3c.org) provides a good generic definition of a Service-Oriented Architecture (SOA) as a set of components that can be invoked and whose interface descriptions can be published and discovered. A web service is a specific instance of a component (or components) that has a public interface defined and described in XML and that other systems can discover and use by passing messages transported via existing internet protocols [3].

On the surface, the characteristics of SOA can be found in other distributed computing systems, but they have substantial differences. The significant difference between them is that each service (component) is loosely coupled, that is to say, the component that provides a service is an autonomous system. The communication from one service to another or from the service-requester to the service-provider are all completely based on open standard protocols, thus, the technical implementation of each component can be different. So, SOA is an architecture whose application is platform independent and technology independent, and this is the embodiment of its core value.

In a basic SOA model, there are three roles [4]:

- Service provider.

- Service broker.

- Service requester.

These characteristics of SOA help it to gain the advantage that other similar systems cannot achieve: in completely off coupling systems, sharing information is easy to implement.

\section{Emergency Vehicles Information System}

\subsection{Compositions}

Emergency vehicles information system is a comprehensive platform which is in connection with environmental pollution accidents. Generally, it should include the following several subsystems [5]:

- Data center and data exchanging subsystem. This part provides the basic data and professional data such as environment protection departments' data, GIS (Geographic Information System) data, resource data etc. It is the foundation of other subsystems.

- Forecasting warning subsystem. This part provides the functions to analysis the accident developing trend and forecast the pollution distribution in time and space dimensions and the occurrence probability of specific events.

- Digital response plan subsystem. This part generates the emergency treatment plan and implementation solution according to basic data and forecast data.

- Decision supporting subsystem. It can integrate many variable factors and contradictory factors to provide a decision supporting.

- Commanding and scheduling subsystem. This is used to issue instructions to coordinate personnel dealing with the pollution.

- Feedback subsystem. It can be used to feedback data which is helpful to fix command giving out by the commanding and scheduling subsystem.

- Disaster evaluation subsystem. Using to evaluate the economic loss caused by the disaster.

- Training and drilling subsystem. Using to train the monitoring personnel and to exercise monitoring business. 


\subsection{System Framework}

The current system framework of the emergency vehicles information system is shown in Fig. 1. There are two cities presented in the figure.

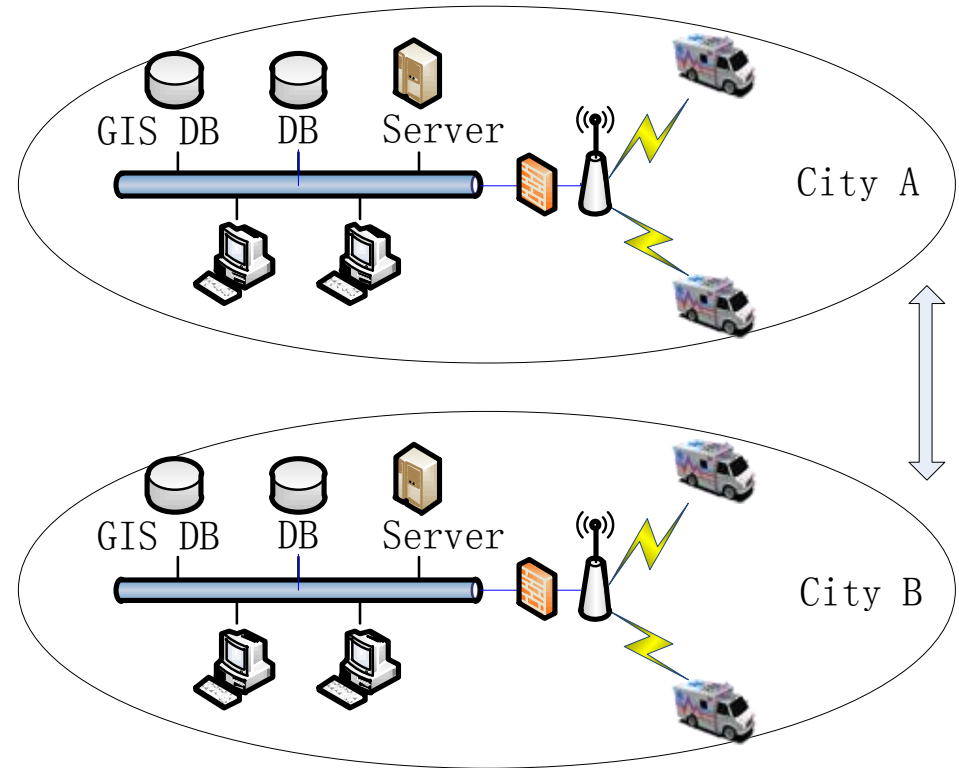

Fig. 1. The system framework of the EVIS

Fig. 1 illustrates how the emergency vehicle connect to the server where contain the core business processing procedure. All the business logic implementations and the data used to do the monitoring work are in the local area network system. An emergency vehicle information system's 8 modules presented in the previous section are distributed in several DBs and servers. When the monitoring personnel worked with the vehicle, they deal with business through a wireless network which can be implemented by $3 \mathrm{G}$ mobile communication or GPRS service in $2 \mathrm{G}$.

In this scheme, the content in the vehicle is just a client to request the server. The server will process all the important businesses, and the subsystems' data is static since the data between several cities cannot be shared. Thus, the data center and data exchange subsystem, the digital response plan subsystem and other subsystems cannot actively integrate data to give out a more accurate result to help the personnel's' work. And this is a crucial respect, because the experiences and older cases' information are significantly important for the current accident.

\section{A Service-Oriented Architecture Framework}

\subsection{System Framework based on SOA}

Since vehicles in the same city or in the different cities cannot share data which is helpful to monitoring the accident, we provide a SOA-based framework to deal with the problem.

As seen in the previous section, the subsystems should work together. In an emergency vehicles information system, the chemical information, risk source information, pollution monitoring cases information, digital 
response plan information, experts information, are the basis in the data center. And all above information can be shared between different accidents or different cities. In a real work, when the monitoring personnel finish his work, he could have not uploaded the real-time data to the server yet, and the experiences have not shared with other people too. And also the pollution monitoring cases information and the digital response plan information. So the vehicle should have a mechanism to share the information in the real time.

Unlike the traditional system framework, the vehicle in the system is not just a client at all. A very important reason why the data cannot be shared is that, the vehicle is just a requester. When the personnel use the system to help them to do the business work, they call the server through the client in the vehicle. Not only the vehicle in different cities but also the ones in the same city cannot share information. So, in the SOA-based framework, the vehicle plays a role like the server.

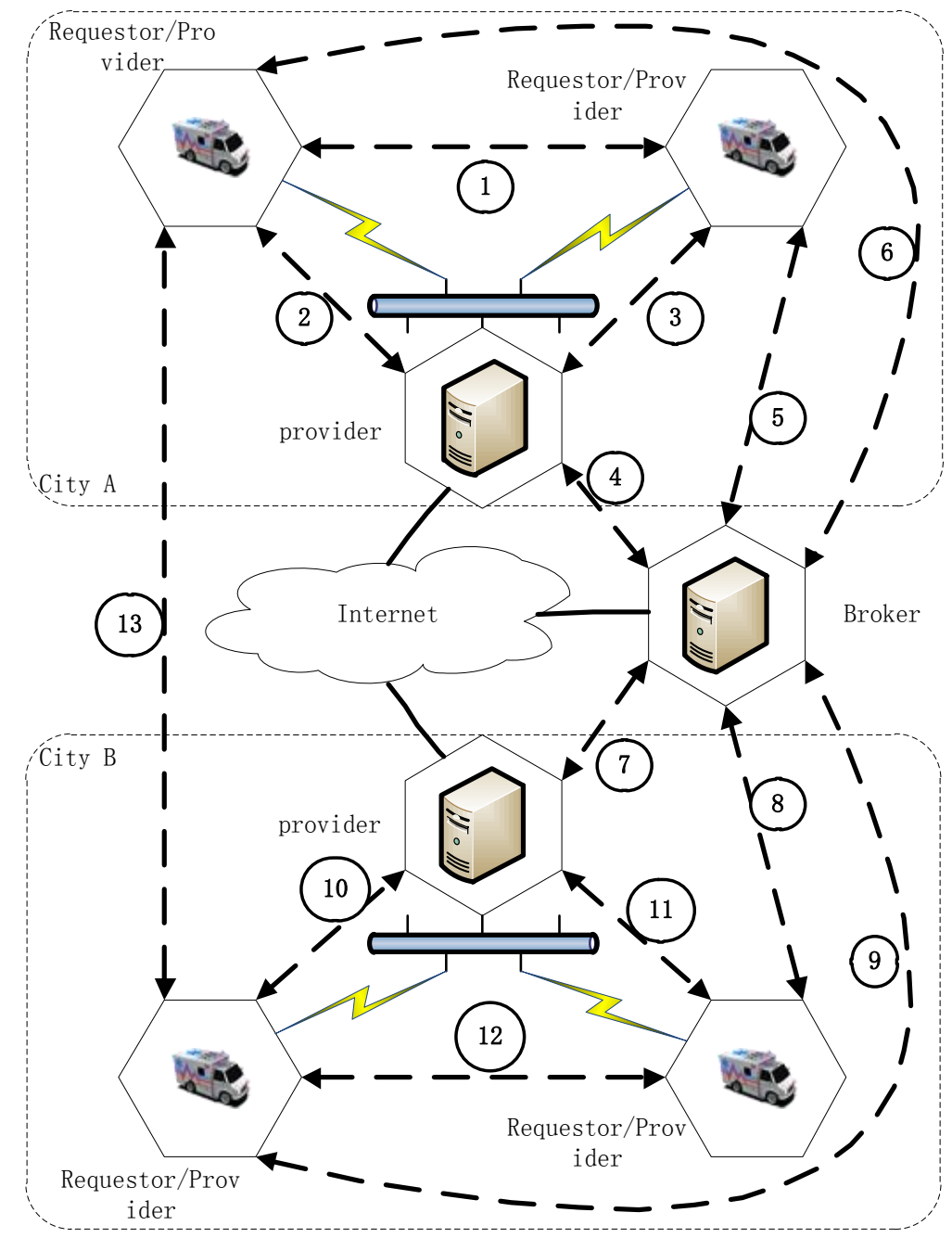

Fig. 2. The SOA framework for the EVIS

Fig. 2 shows the framework. In the framework, we explain how the vehicles in the same city or in different cities share information. There are some points: 
- Each part can work alone as an autonomous system. The server provides services or data to the vehicles, the vehicles request the services and data provided by the server and also provide services to other part.

- Each part is in the network, they can connect to each other. The vehicles connect the network by a wireless communication, the server and the vehicles are in a LAN. The servers in different cities and the broker are connected by the internet.

- The broker is an intermediary. Each part except the broker can provide services and each vehicle can request services from the providers. The services provider register services in the broker and the services requester find them in the broker.

- The broker can belong to any city, or as a lonely one.

In section III, we gave out the composition of an emergency vehicles information system. In the SOA-based framework, the data center and data exchanging subsystem, the forecasting warning subsystem, the decision supporting subsystem, the commanding and scheduling subsystem are distributed in the server; the digital response plan subsystem, the feedback subsystem, the disaster evaluation subsystem, the training and drilling subsystem are distributed in the vehicle.

The numbers in Fig. 2 represent the data flows and the operation flows. The service providers register and publish services in the service broker, and the service requesters find the services also in the broker. Then the requester binds the service which is needed to the provider.

The numbers 4,5,6,7,8,9 in Fig.2 show that each component in the framework publishes services in the broker. So the broker has storage of all the subsystem's services. The numbers 5,6,8,9 show that the vehicles find services from the broker. The numbers $1,2,3,10,11,12,13$ show that service requesters use services from the providers after binding the services.

\subsection{Services Modeling}

The 8 subsystems' components have several services to support the whole emergency vehicles information system. According to [6][7][8], here we introduce the services that the forecasting warning subsystem provides.

1) GIS Service. To provide a intuitive presentation of the pollution diffusion simulation, the GIS(Geographic Information System) is needed.GIS service provides the functions like getting map, getting layer, getting table, routing, encoding and so on.

2) Diffusion Model Service. The service provides the functions like Gaussion Model Formula, air concentration computing, contour line point computing.

3) Meteorological Service. The service provides the functions like getting sun elevation angle, getting sun inclination angle, getting sun radiation level, getting air steady level.

4) Realtime Data Service. The service provides the functions like gett data of the pollution, getting parameters, getting data unit, getting monitoring time, getting pollution id, getting monitoring position, getting sampling rate.

\subsection{Services Discovery and Composition}

After all components register their services in the service broker, the vehicle can find a matched service from the broker. For example, to forecast the pollution diffusion, it should find Diffusion Model Service and GIS Service. The GIS Service can be found which provided by the server component, then the vehicle bind the service to the server. However, the Diffusion Model Service needs some services to help it to finish the task. In this case, the services should be composited. The Diffusion Model Service, the Meteorological Service and the Real-time Data Service would composite to a new one which can provide matched services. 


\subsection{An Example of Business Processing}

Fig. 3 shows the representation of a business processing procedure that how to simulate the air pollution diffusion.

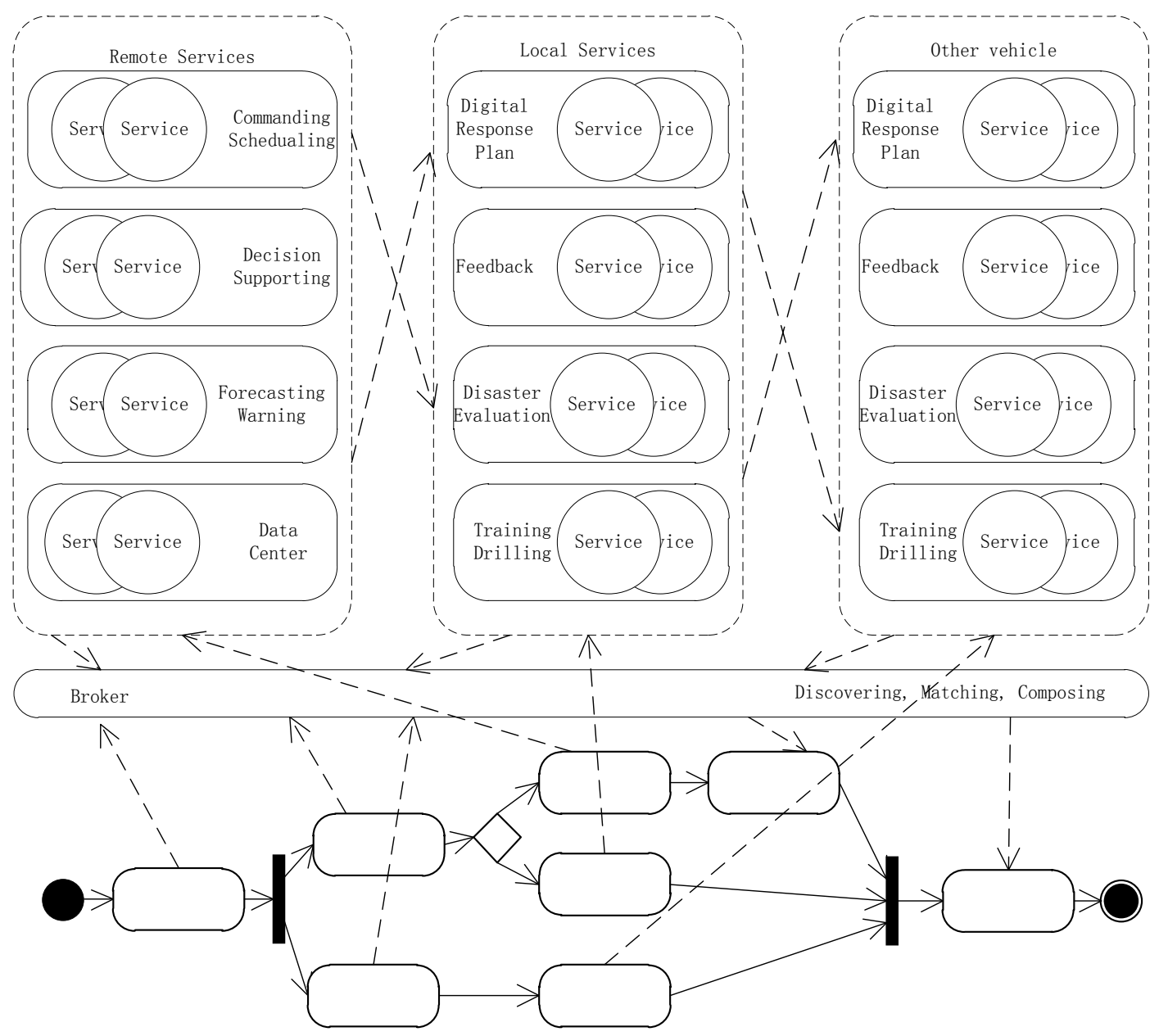

Fig. 3. An example of Business Processing

In the field of environment monitoring, the processing of pollution diffusion simulation is a hard work since there are plenty of parameters in the processing procedure and the factors are always changeful. So monitoring personnel should work with not only the data that can get by themself but also the data that get from other personnels who were in the different vehicles or in the different cities.

The procedure of pollution diffusion simulation should supported by the GIS service, the meteorological service, the diffusion model service, and the real-time data service. The vehicle system finds the services from the services broker. A situation would happen that the meteorological service in local vehicle do not matched the requirement, and the GIS service is not in the local vehicle. Then the vehicle system would find another 
service that is suitable. As shown in Fig.3, the services from the local vehicle, the remote server and other vehicles would be composed to another and a service would invoke other services to match the requirement.

The components that request the services would send SOAP messages to the service providers after binding services. The meteorological service and the real-time data service supply parameters to the diffusion model service, and it would feedback the diffusion information after computing. All these information then send to the GIS service by SOAP messages. The GIS service component would draw some curve on the GIS map. After that, the service of pollution diffusion simulation is invoked completely.

\section{Conclusion}

This paper addressed the application of SOA in the field of emergency vehicle information system. The traditional framework was discussed. Particularly, we proceed with the composition of a standard emergency vehicle system. The core of this paper is to propose a Service-Oriented Architecture Framework for emergency vehicles information system. The details of the framework were discussed and also an example of business processing in the framework was proposed.

\section{References}

[1] Do van Thanh, Ivar Jorstad, "A Service-Oriented Architecture Framework for Mobile Services," Advanced Industrial Conference on Telecommunications/Service Assurance with Partial and Intermittent Resources Conference/E-Learning on Telecommunicatins Workshop, July 2005, pp. 65-70, doi:10.1109/AICT.2005.14.

[2] Michael p. Papazoglou, Paolo Traverso, "Service-Oriented Computing: State of the Art and Research Challenges," Coputer, vol.40, Nov. 2007, pp. 38-45, dio:10.1109/MC.2007.400.

[3] Nicolas Gold, Andrew Mohan, Claire Knight, Malcolm Munro, "Understanding Service-Oriented Software," Software, Vol.21, April 2004, pp.71-77,doi:10.1109/MS.2004.1270766.

[4] Ning Gu, Jiamao Liu, Xiaolu Chai, Web Services Principles, 1st ed., Beijing: China Machine Press, 2006, pp.69-90. (in Chinese)

[5] Chaoyang Fu, Qinxian Jin, "Research on the General Framework and Composition of Environment Emergency Management Information System," Environmental Monitoring in China, vol.23, Oct. 2007, pp. 82-86. (in Chinese)

[6] Thomas Erl, SOA: Principles of Service Design, 1st ed., London: Pearson Education, 2008, pp.18-69.

[7] Paul C. Brown, Implementing SOA: Total Architecture in Practice, 1st ed., London: Pearson Education, 2008, pp.63-158.

[8] Zhiyi Ma, Hongjie Chen, "A Service-Oriented Architecture Reference Model," Chinese Journal of Computers, vol.29, July 2006, pp. 1011-1019. (in Chinese) 